Supplementary Information

for

\title{
Moving Kinetics of Nanocars with Hydrophobic Wheels on Solid Surfaces at Ambient Conditions
}

\author{
Submitted to J. Phys. Chem. C for publication
}

Fang Chen ${ }^{\mathrm{a}}$, Víctor García-López ${ }^{\mathrm{b}}$, Tao Jin ${ }^{\mathrm{a}}$, Bhanu Neupane ${ }^{\mathrm{a}}$, Pin-Lei E. Chu ${ }^{\mathrm{b}}$, James Tour ${ }^{\mathrm{b}, *}$, Gufeng Wang ${ }^{\mathrm{a}, *}$

${ }^{a}$ Department of Chemistry, North Carolina State University, Raleigh, NC 27695-8204

${ }^{\mathrm{b}}$ Department of Chemistry, Rice University, Houston, TX 77005

Address all correspondence to:

James Tour, Department of Chemistry, Rice University, Houston, TX 77005. E-mail: tour@rice.edu

Or

Gufeng Wang, Department of Chemistry, North Carolina State University, Raleigh, NC 276958204. Tel: (919) 515-1819; E-mail: gufeng_wang@ncsu.edu 


\section{MOVIES}

All movies collected at 1 frame/min and played at 15 frames/second (900 times faster). The image areas in all movies are all $20 \times 20 \mu \mathrm{m}^{2}$.

Movie 1. Freshly prepared nanocar molecules diffusing on hydroxylated glass surface.

Movie 2. Nanocar molecule diffusion on glass surface after $3 \mathrm{~h}$.

Movie 3. Nanocar molecule diffusion on glass surface after $24 \mathrm{~h}$.

Movie 4. Freshly prepared nanocar molecules diffusing on PEG-modified glass surface.

Movie 5. Nanocar molecule diffusion on PEG-glass surface after $12 \mathrm{~h}$.

Movie 6. Nanocar molecule diffusion on PEG-glass surface after $72 \mathrm{~h}$. 


\section{Hydrocarbon-like molecule deposition rate on clean glass surface in sticky-spots model}

In the sticky spots model, it is assumed that the organic molecules in the air will adsorb on the glass surface and form "sticky" sites. There are many "sticky" sites distributed randomly on the glass surface. The nanocar molecule will diffuse slower on these sites because of higher activation energy needed. Thus, whenever a nanocar molecule encounters such a sticky site, it will be retained on the site for a much longer time, just like a macroscopic car met a "sticky spot". The nanocar will have "on time" $t_{\text {on }}$ when it is on the sticky site, and "free time" $t_{\text {free }}$ when it is freely diffusing on glass surface. In each "on" and "free" cycle, the molecule moving distance could be described as:

$$
L^{2}=4 D(0) t_{\text {free }}
$$

where $L$ is the step size within a "on" and "free" cycle; $D(0)$ denotes the diffusion coefficient of a nanocar on a clean glass surface assuming no adsorbate on the surface. Generally, we deem that the diffusion coefficient measured at the fresh surface can be viewed as $D(0)$.

Practically, the apparent diffusion coefficient $D(t)$ after aging time $t$ is determined:

$$
L^{2}=4 D(t) t_{\text {total }}
$$

where $t_{\text {total }}$ is the total cycle time. Since in each cycle, the nanocar will meet a sticky site once, the sticky site density $(n)$ can be estimated:

$$
L^{2} \cdot n=1
$$

Thus, from S1 and S3, we obtain:

$$
t_{\text {free }}=1 / 4 n D(0)
$$

From S1 and S2, the ratio of real diffusion coefficient and apparent diffusion coefficient could be estimated as:

$$
\frac{D(0)}{D(t)}=\frac{t_{\text {total }}}{t_{\text {free }}}=1+\frac{t_{\text {on }}}{t_{\text {free }}}=1+4 n D(0) t_{\text {on }}
$$

With time increasing, more and more organic molecules will be adsorbed on the surface and form more sticky sites. Assuming that the number of sticky sites increases linearly with respect to the aging time:

$$
n=k t
$$

where $k$ is the adsorption rate of organic molecules; $t$ is aging time. The nanocar diffusion kinetics can be described as a linear equation:

$$
\frac{D(0)}{D(t)}=1+4 D(0) t_{o n} k \cdot t
$$

The slope contains information about $D(0)$, the on time $t_{o n}$, and the adsorption rate $k$.

(1) This relationship is qualitatively consistent with the experimental data both for hydroxylated glass surface and the PEG-modified surface (Supporting Figure 4). Importantly, the slope for the glass surface is $\sim 4$ times that of the PEG modified surface, indicating the adsorption rate is much faster on the glass surface than on the PEG modified surface.

(2) More importantly, the adsorption rate of the hydrocarbon-like molecules can be estimated. From the slope for the glass surface $(0.111 / \mathrm{h})$ and the $D(0) 7.6 \times 10^{-16} \mathrm{~m}^{2} / \mathrm{s}$, we can estimate the $t_{\text {on }} \cdot k$ to be $\sim 36(\mathrm{~s})\left(\# / \mu \mathrm{m}^{2} / \mathrm{h}\right)$. If we further assume $t_{\text {on }}$ to be on a time scale from $\mathrm{ms}$ to second (1 $\mathrm{ms} \sim 1 \mathrm{~s})$, we can find that $k$ to be $36 \sim 36000$ molecules $/ \mu \mathrm{m}^{2} / \mathrm{h}$. This value is consistent in the order of magnitude to the values reported in the literature. For example, Chasse et al. reported 
$16 \%$ of carbon coverage on Ar-implanted silicon surface with $200 \mathrm{~h}$ aging time in air, which gives an adsorption rate of $\sim 0.08 \%$ per hour. ${ }^{1}$

These estimates may not necessarily prove that our SSM model is correct but it shows that our model is self-consistent and possible.

\section{Reference}

(1) Chasse, M.; Ross, G. G. Effect of Aging on Wettability of Silicon Surfaces Modified by Ar Implantation. J. Appl. Phys. 2002, 92, 5872-5877. 


\section{Supporting Figures}

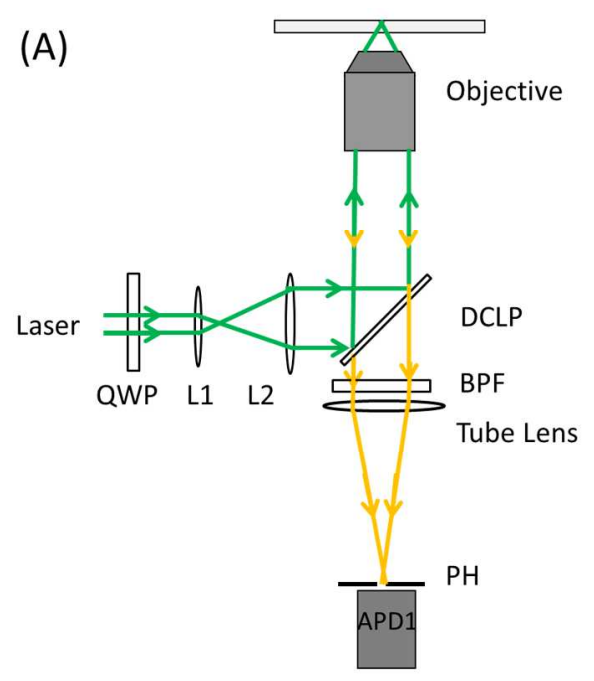

(B)

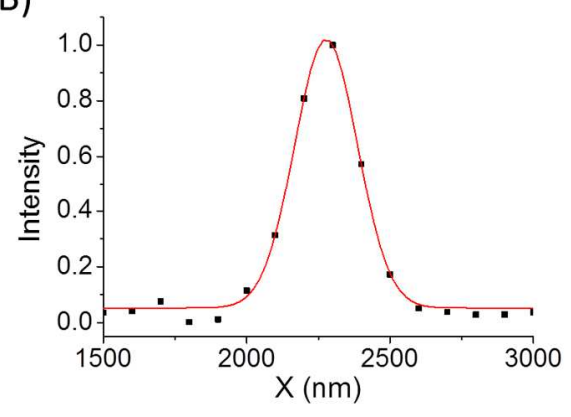

Supplementary Figure 1. Schematic of the home-built confocal single molecule fluorescence microscope. (A) Schematic of the confocal setup. (B) Intensity profile of one selected molecule. The FWHM peak maximum is $260 \mathrm{~nm}$. 

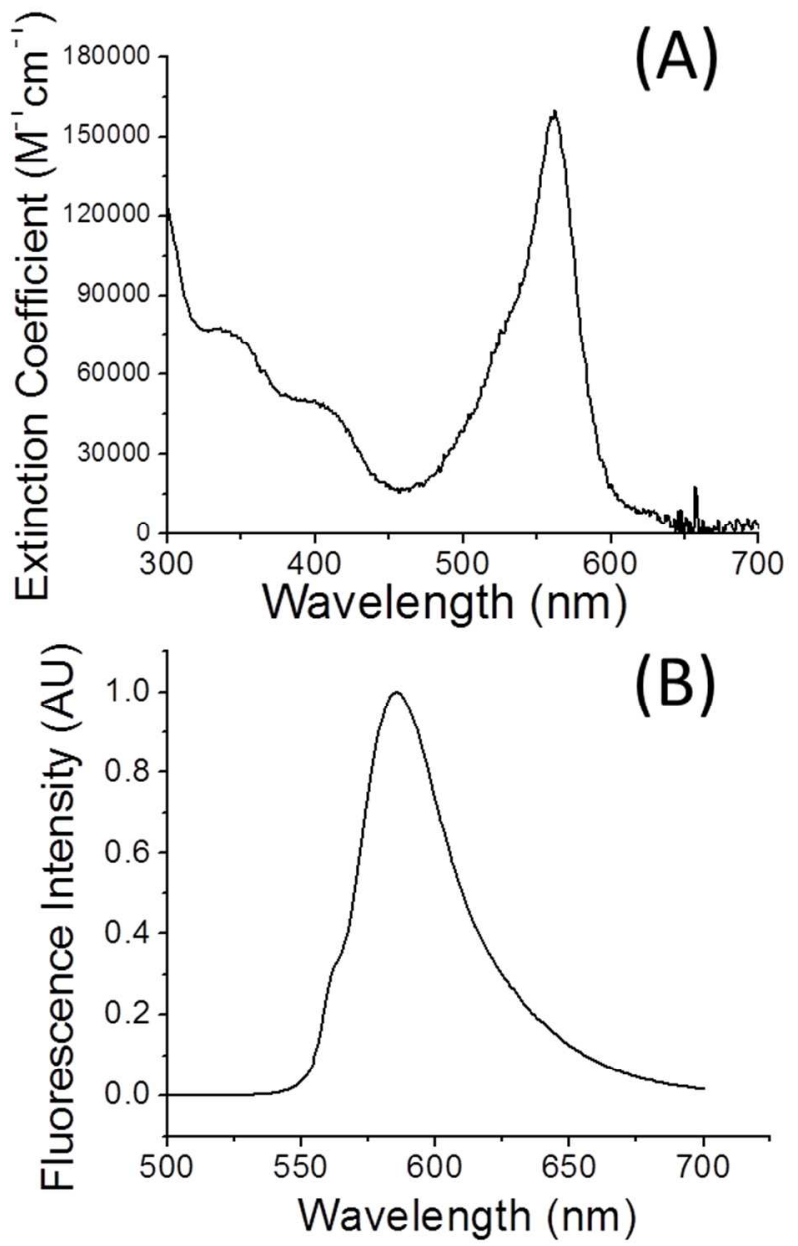

Supporting Figure 2. UV-VIS and fluorescence spectra of 4-wheeled adamantane nanocars. (A) UV-Vis. (B) Fluorescence. Spectra were taken in $2.0 \mu \mathrm{M}$ solution in acetonitrile. 
(A)

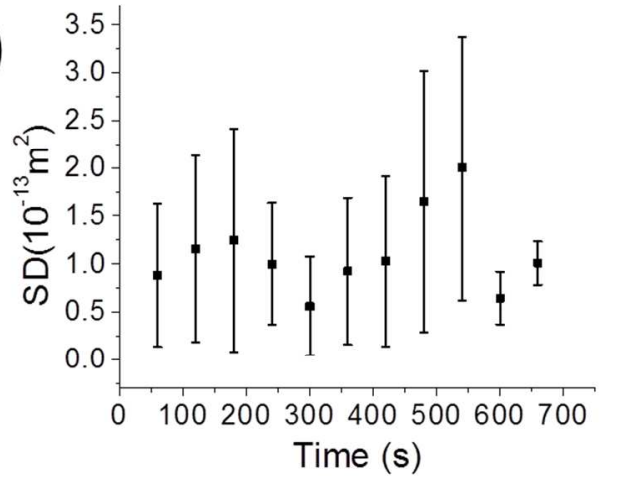

(B)

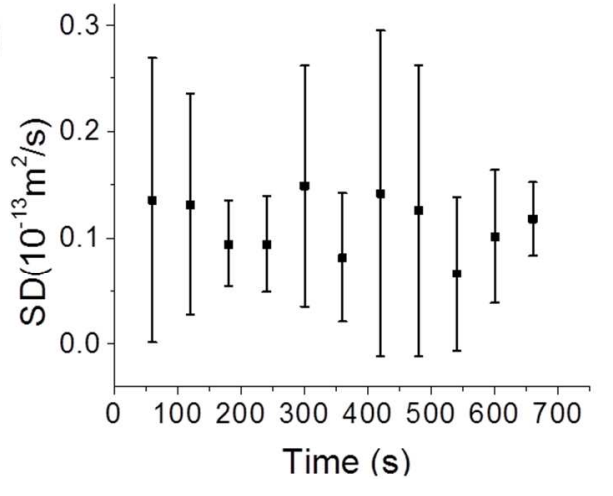

Supporting Figure 3. MSD as a function of time. (A) Sample aged for 3 hours. (B) 12 hours. The non-linearity of the MSD curve possibly suggests that the surface diffusion became nonBrownian. 

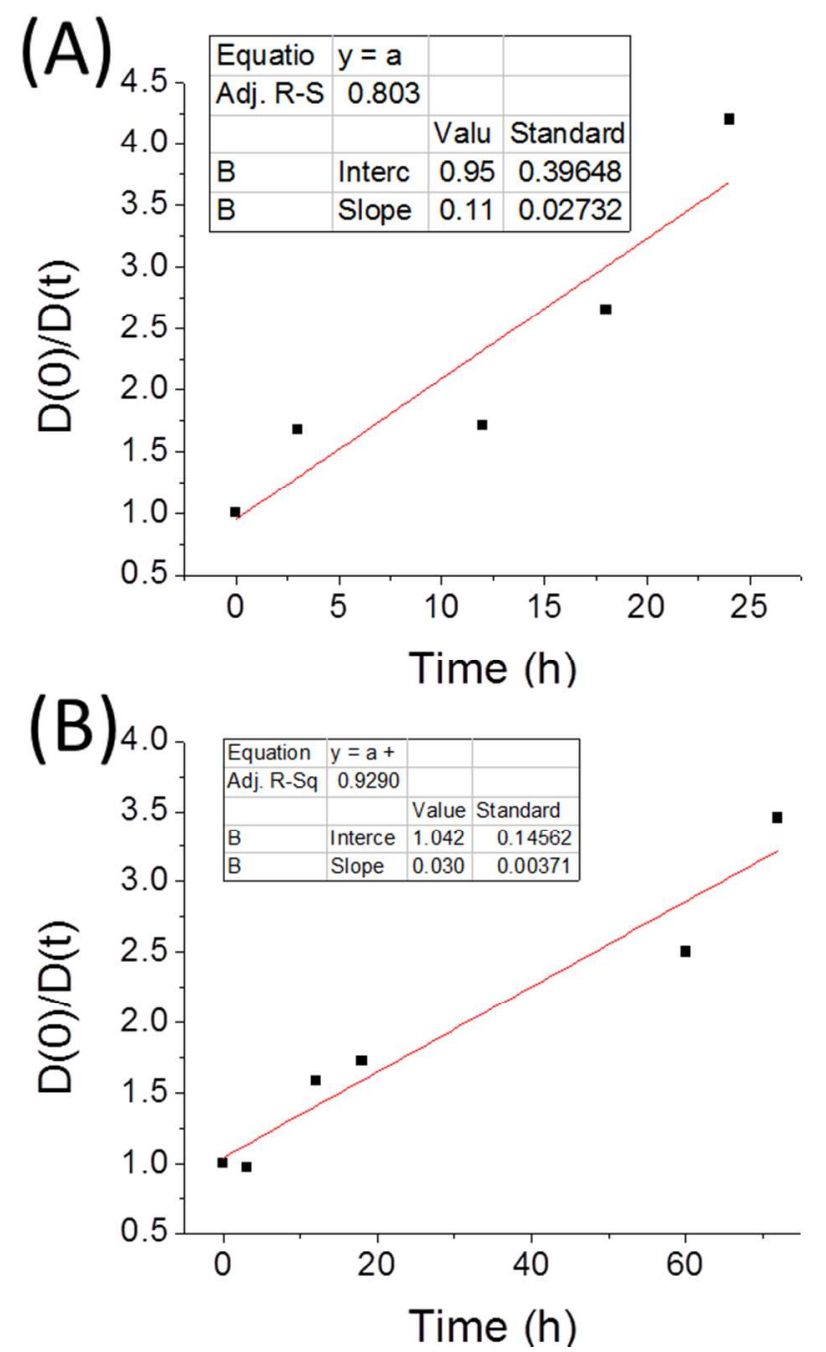

Supporting Figure 4. Ratio between $D(0)$ and $D(t)$. (A) Hydroxylated glass surface. (B) PEGmodified glass surface. 